Braz J Med Biol Res, April 2012, Volume 45(4) 337-348

doi: $10.1590 / \mathrm{S} 0100-879 X 2012007500038$

On the relationships between ultrasonic calling and anxiety-related behavior in rats

R.K.W. Schwarting and M. Wöhr

The Brazilian Journal of Medical and Biological Research is partially financed by

\section{贵CNPq}

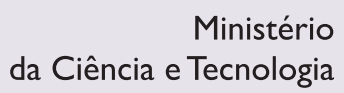

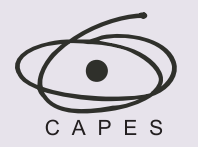

Ministério da Educação
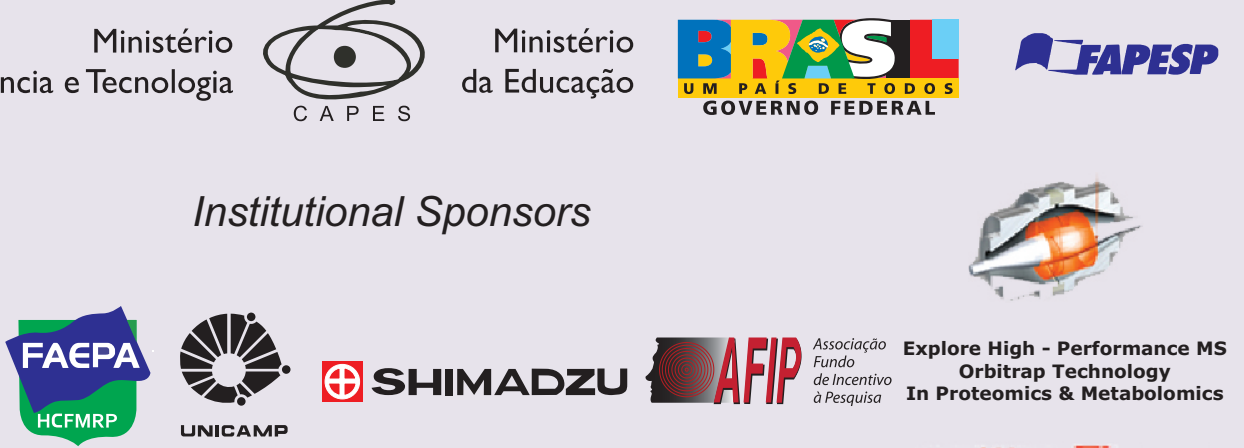

Institutional Sponsors
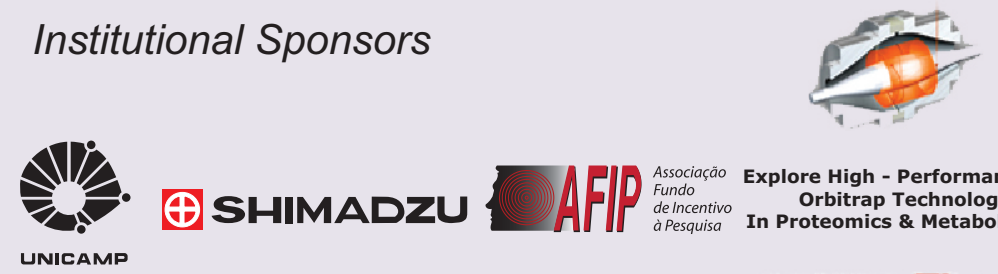

๑ SHIMADZu UNICAMP

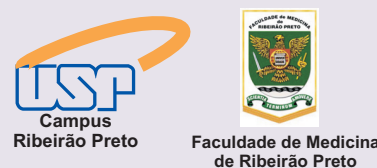

aculdade de Medicic
de Ribeirão Preto 


\title{
On the relationships between ultrasonic calling and anxiety-related behavior in rats
}

\author{
R.K.W. Schwarting and M. Wöhr
}

Experimental and Physiological Psychology, Philipps-University of Marburg, Marburg, Germany

\begin{abstract}
In the present review, the phenomenon of ultrasonic vocalization in rats will be outlined, including the three classes of vocalizations, namely $40-\mathrm{kHz}$ calls of pups, and $22-$ and $50-\mathrm{kHz}$ calls of juvenile and adult rats, their general relevance to behavioral neuroscience, and their special relevance to research on anxiety, fear, and defense mechanisms. Here, the emphasis will be placed on 40- and 22-kHz calls, since they are typical for various situations with aversive properties. Among other topics, we will discuss whether such behavioral signals can index a certain affective state, and how these signals can be used in social neuroscience, especially with respect to communication. Furthermore, we will address the phenomenon of inter-individual variability in ultrasonic calling and what we currently know about the mechanisms, which may determine such variability. Finally, we will address the current knowledge on the neural and pharmacological mechanisms underlying 22-kHz ultrasonic vocalization, which show a substantial overlap with mechanisms known from other research on fear and anxiety, such as those involving the periaqueductal gray or the amygdala.
\end{abstract}

Key words: Ultrasonic vocalization; Anxiety; Fear; Communication; Social behavior

\section{Introduction}

Rats, like many other rodents including mice, can vocalize in the sonic and ultrasonic range, that is, within or above the human hearing range, which has its upper limits at about $20 \mathrm{kHz}$. The rats' sonic calls or squeals, which are not a topic of this review, range from 2 to $4 \mathrm{kHz}$, and are emitted when a rat is confronted by a predator. They seem to serve as a threat signal directed at the predator, signaling that the rat is prepared for a defensive attack (for a review, see Ref. 1).

Regarding the ultrasonic vocalizations (USV) of rats, our knowledge is based on the pioneering article of Anderson (2), who measured distinct ultrasonic signals from rats in their cages. Anderson did not relate them to any eliciting stimuli or internal states, but suggested that they may serve for communication between rats or for echolocation. Since this initial observation, USV have been intensively investigated, for example with respect to their possible functional role(s), and the underlying physiological and pharmacological mechanisms. Thus, a substantial amount of experimental evidence has been gathered, especially during the 70-80 decades of the last century (3), although more recently USV have received renewed and increasing scientific attention.
This 'revival' is partly due to new and refined research hypotheses, and also to the fact that sophisticated equipment has meanwhile become commercially available (for technical details, see Ref. 4), which permits the analysis of vocalizations in great detail (i.e., spectrographically). Also, such equipment can produce USV, for example in behavioral playback studies, that is, calls of a sender can nowadays not only be measured with great sophistication, but they can also be presented and manipulated to analyze their critical signal features in the recipient (for an example, see Ref. 5). These developments have led to the status quo of pertinent research where USV analysis encompasses a number of basic and translational research fields, including auditory physiology, communication, emotion, motivation, social neuroscience, and various preclinical animal models including anxiety, depression, autism, addiction, and Parkinson's disease (for a review, see Ref. 1).

Currently, we know that rat USV can encompass the whole range of 20 to about $100 \mathrm{kHz}$ (2). Within this range, which includes call fundamentals and harmonics, three distinct USV classes can be defined. These are termed 40-, 22- and $50-\mathrm{kHz}$ calls, which serve as situation-dependent

Correspondence: R.K.W. Schwarting, Experimental and Physiological Psychology, Philipps-University of Marburg, Gutenbergstr, 18, 35032 Marburg, Germany. Fax: +64-2128-23610. E-mail: schwarti@staff.uni-marburg.de

Presented at the III Fórum em Neurobiologia do Estresse, Araraquara, SP, Brazil, September 8-10, 2011.

Received November 30, 2011. Accepted March 8, 2012. Available online March 23, 2012. Published April 9, 2012. 
and affect-related signals (for reviews, see Refs. 6-8) (Figure 1). In general, rats probably produce USV during exhalation and in a whistle-like manner (9), for which laryngeal function seems to be critical. Also, they are able to detect such ultrasonic signals since their auditory system includes tonotopic representations for USV frequencies at several neural levels. The ultrasonic signals themselves are suitable for short-range and directional communication, since ultrasonic signals are attenuated by atmospheric pressure and are scattered by small objects in the environment, like grass, etc., especially if calls are of higher frequency and complex frequency modulation. Furthermore, USV even seem to work in tunnel systems, one of the rats' natural habitats. Overall, the calls may be suitable to be detected by nearby conspecific rats, but less so by more distant predators, some of which are also able to detect ultrasonic calls (for a review, see Ref. 1).

Out of three known classes of rat USV, 40- and 22-kHz calls will be outlined in this review in more detail, since they are related to aversion and seem to be part of the species' defense mechanism, that is, they occur in response to immediate and potential environmental threats, whereas $50-\mathrm{kHz}$ calls, which are related to appetitive behavior, will be addressed only shortly for the sake of completeness.

\section{0-kHz calls}

The $50-\mathrm{kHz}$ class comprises calls that are emitted by juvenile and adult rats. These calls are of rather short duration (i.e., around 20-100 ms) and show considerable variability in frequency modulation $(35-70 \mathrm{kHz})$. They are typical for appetitive social interactions and seem to promote them, including their induction and maintenance. Thus, juvenile rats emit $50-k H z$ calls during rough-and-tumble-play or when being tickled playfully by a skilled experimenter (10-13). Also, adult rats show high rates of $50-\mathrm{kHz}$ USV in anticipation of or during mating (14), feeding, electrical stimulation of the brain (15), or addictive drugs, especially psychostimulants (16-18). On the other hand, aversive stimuli like cat scents (6), bright light (10), or presence of a footshock cue (16) can inhibit 50-kHz calling in otherwise rewarding situations.

The following and major sections of this review are devoted to calls, which are typical for aversive situations, namely $40-\mathrm{kHz}$ calls in pups, and $22-\mathrm{kHz}$ calls in juvenile and adult rats.

\section{0-kHz calls}

These calls, which are emitted by rat pups, are relatively short, i.e., they last about $80-150 \mathrm{~ms}$, and actually range around $30-65 \mathrm{kHz}$. Pups emit them in response to a potentially distressing situation, namely separation from mother and litter (for reviews, see Refs. 1,19). Call likelihood increases around postnatal days (PND) 3-5; maximal call numbers are reached during about PND 5-10, and calls disappear again until around PND 21. The critical stimulus for their occurrence is probably not just the separation from mother and litter, but the resulting drop in ambient temperature. Thus, isolated pups, which cannot yet maintain their body temperature, do not call or call considerably less when tested under temperature conditions similar to that of the nest. Therefore, precise control of ambient temperature is a must in studies of pup USV.

The pup's acoustic signal is of major survival value, since it leads to retrieval behavior in the mother (20). This function can be specifically tested using a playback approach, where a dam is exposed to an acoustic environment, for example, a radial maze-like apparatus, with no pups present but with their calls provided by a specific ultrasonic loudspeaker (Figure 2). We have used this approach, for example, to present natural $40-\mathrm{kHz}$ calls versus artificial $40-\mathrm{kHz}$ sine-wave tones (21). The test mothers typically responded with behavioral activation and approach. This activation was stimulus-dependent, since arms in front of the stimulus source were preferred only during playback of $40-\mathrm{kHz}$ calls but not during sine-wave signals of the same frequency level. Such a playback approach has the specific advantage that the effectiveness of a single feature (here acoustic) of an otherwise complex social stimulus, namely 'pup', can be tested and varied systematically to investigate its effectiveness in the recipient. Our findings

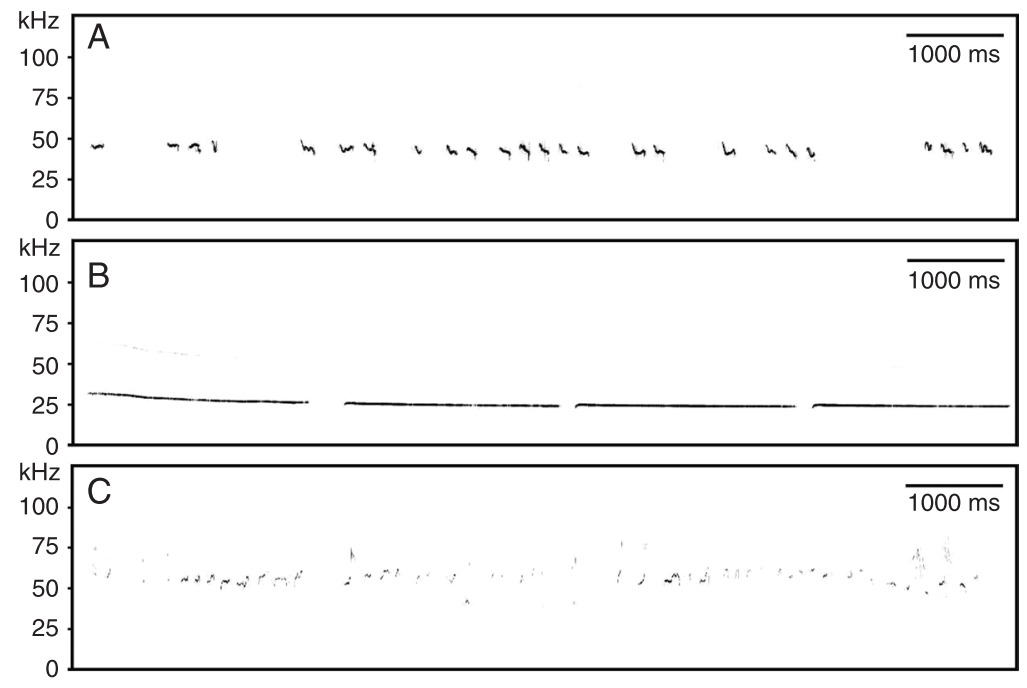

Figure 1. Types of rat ultrasonic vocalizations (USV). A, Isolation-induced $40-\mathrm{kHz}$ USV emitted by an 11-day-old male Wistar rat after separation from mother and littermates. $B$, Low-frequency $22-\mathrm{kHz}$ USV emitted by a 3-month-old male Wistar rat during fear conditioning. $C$, High-frequency $50-\mathrm{kHz}$ USV emitted by a 3-month-old male Wistar rat searching for conspecifics. 
support the hypothesis that specific call parameters, like the extent of frequency modulation, are critical to induce approach and retrieval behavior, probably since alternating frequency sweeps can be detected more easily than a steady sound.

Often, these calls have been considered in the literature as a correlate (and thus experimental measure) of a presumptive state of anxiety or distress, especially since they can be reduced or blocked by anxiogenic drugs like benzodiazepines, 5-hydroxytryptamine ${ }_{1 \mathrm{~A}}\left(5-\mathrm{HT}_{1 \mathrm{~A}}\right)$ agonists, and selective serotonin reuptake inhibitors (SSRIs) (see, e.g., Refs. 22-24). Also, it was shown that rats bred over several generations for high rates of isolation-induced calling as pups showed more anxiety-related behavior in adulthood than animals bred for low call rates (25). Furthermore, animals selectively bred for an adult anxious phenotype (as gauged by several adult measures of anxiety) were found to show more isolation-induced vocalizations as pups than the less anxious phenotype (26-28).

The anxiety hypothesis of pup vocalizations, however, was questioned by Blumberg and Sokoloff (29), who suggested that these calls actually might be "acoustic byproducts of a physiological maneuver that maintains cardiopulmonary homeostasis" in response to the cooling challenge induced by isolation (cited from Ref. 30). Several lines of evidence support this physiological hypothesis and argue against the anxiety hypothesis.

For one, various drugs or neurotransmitters apart from anxiolytics can reduce pup calling, including morphine, enkephalin, dopamine receptor agonists, cocaine, muscarinic agonists, norepinephrine, yohimbine, NMDA agonists, corticotrophin-releasing factor (CRF), and cannabinoids.
Norepinephrine, for example, is a $\beta$-adrenoceptor agonist with anxiogenic effects in adults, yet it suppresses calls in pups. The a2-agonist clonidine, on the other hand, is anxiolytic in adults, but promotes pup calls rather than suppressing them (according to Ref. 24). Secondly, rather high benzodiazepine doses are required for an effect on pup vocalization. Since these doses are also sedative, a specific anxiolytic interpretation in case of pups is doubtful. Thirdly, Middlemis-Brown et al. (30), using lesion techniques, showed that brainstem structures (up to the midbrain) are sufficient for pup vocalization, that is, a presumptive anxiety response could be elicited despite the lack of major forebrain structures known to be critical for anxiety in adults, such as the amygdala, hippocampus, frontal cortex, etc. Alternatively, one could argue that pup vocalization in response to isolation and the ensuing homeostatic cold threat is a kind of early ontogenetic defensive mechanism with high survival value, which is mediated primarily by brainstem structures, especially the periaqueductal gray (PAG; see also below) and its caudal efferents controlling the motor outputs. This pup defense may occur without a state of anxiety and without a prominent regulation of forebrain structures, which probably come into effect only later during pup development. This assumption is also supported by the finding that hypothermic pups still vocalize when comatose (31).

Fourth, it should be noted that the positive correlations between pup calls and adult measures of anxiety, which were taken as an indication that infant calls might already indicate adult anxiety, were obtained in rats bred either for pup vocalization or for an adult measure of anxiety (25-28). We have repeatedly found that this relationship does not

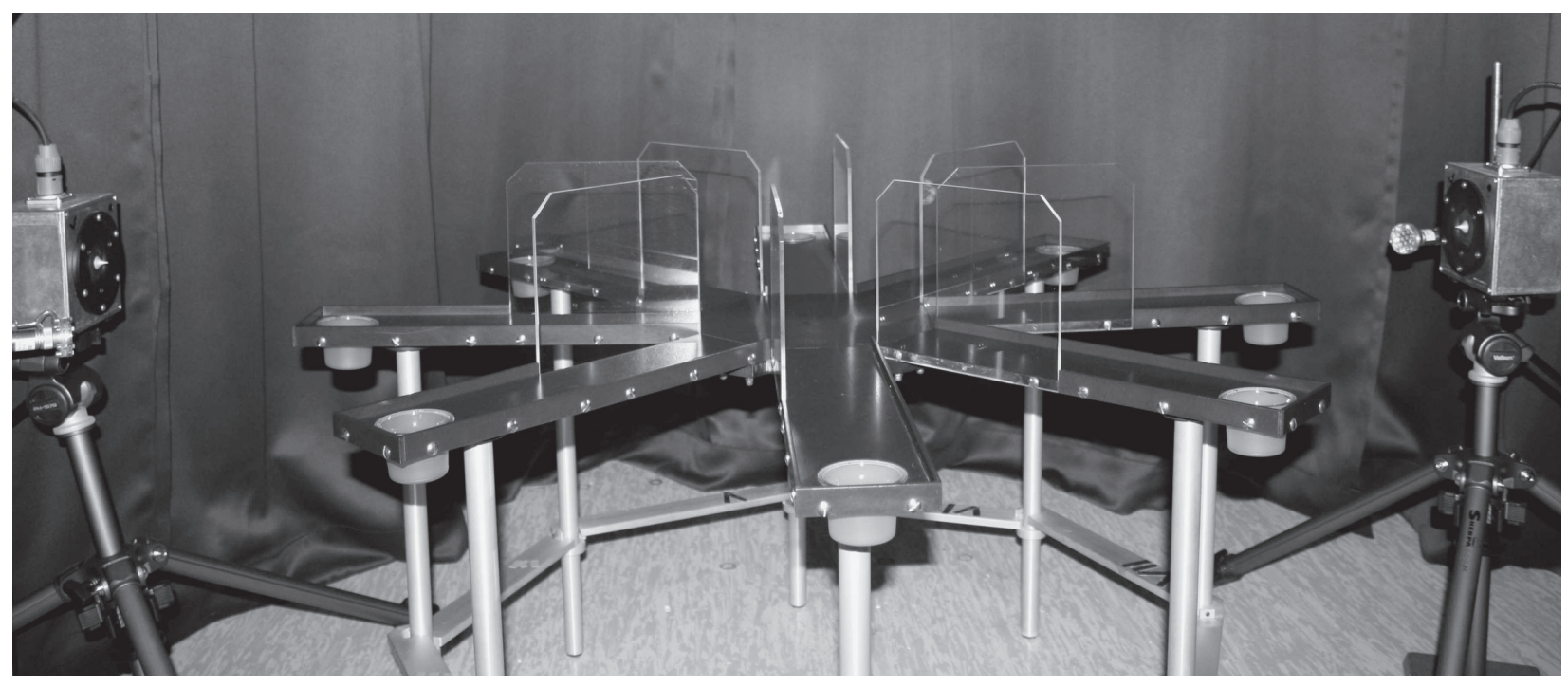

Figure 2. Setup for playback experiments. Elevated eight-arm radial maze (arm length: $40.5 \mathrm{~cm}$ ) equipped with two ultrasonic microphones and two loudspeakers (Avisoft Bioacoustics, Germany). The rat's behavioral responses during playback of ultrasonic stimuli were recorded with a video camera positioned above the elevated eight-arm radial maze. Testing was performed under dim red light. 
hold for unbred Wistar or Sprague-Dawley rats $(21,32,33)$. Thus, and in contrast to the breeding lines, 'regular', i.e., not selectively bred commercial rats showed a positive correlation between pup calls in isolation and adult openarm time in the elevated plus-maze (32). Furthermore, pup calls were negatively correlated with adult $22-\mathrm{kHz}$ calls in a fear-conditioning paradigm $(21,33)$ (Figure 3$)$. This means that rat pups emitting low numbers of calls, supposedly reflecting low levels of distress and anxiety, display an anxiety phenotype in adulthood and vice versa.

The discrepancy between our findings and those obtained with selective breeding lines might be explained by several factors: for one, we used the normal variation in unselected animals and not their extremes, which are used by others to serve as the basis of several generations of breeding. Also, repeatedly bred animals might not simply be a phenotypic exaggeration of their ancestors, but may represent qualitatively new phenotypes, where other mechanisms have come into play through repeated breeding. Thus, it is known from other fields of behavioral research (see, e.g., Ref. 34) that selective breeding based on one behavioral trait can bring a host of other behavioral traits along with it, since the behavior used for breeding is not the only one that is influenced by the genes segregated by the breeding procedure. Nonetheless, such "anxious" breeding lines are of great value when it comes to research on disease models, especially for anxiety as a disease or depression, but such lines may not be assimilable to 'normal' rats. For example, one could argue that emission of ultrasonic calls in early infancy is part of an active defense mechanism, since pup vocalization is often paralleled by, and positively correlated with, behavioral activity like pivoting (for details, see Ref. 21), whereas with further development, call emission becomes part of a passive defense mechanism correlated with immobility or freezing $(4,21)$. In 'normal' rats, these mechanisms may be governed by different neural mechanisms, for example, since pup vocalization is required only during a short developmental period, whereas in the disease models their regulation may have come under the control of the same (hypothetical) mechanism by repeated breeding.

Finally, the phenomenon of maternal potentiation seems to argue against the anxiety hypothesis of pup vocalization. Maternal potentiation means "that the rate of isolation-induced vocalization can be enhanced by recent contact with the dam, but not by other social stimuli that elicit contact quieting, e.g., littermates or home cage shavings" (cited from Ref. 35). If pup vocalization reflected a state of anxiety, this phenomenon would mean that pups become more anxious by having been shortly exposed to their mother, which intuitively seems rather unlikely. Alternatively, the enhanced vocalization response after maternal contact may again reflect an adaptive biological mechanism, which enhances signaling (i.e., pup calls) when external stimuli are provided that the caretaker may be near (as indicated by the previous presence of the mother), whereas vocalization is suppressed when danger stimuli are provided like predators or unrelated male rats (36).

As a whole, the relationship between pup vocalization and anxiety may not be as straightforward as often suggested. Nevertheless, the study of pup vocalizations is of great scientific value, for example, to further our understanding of ontogenetically early defense mechanisms, but great care should be taken when attempting to generalize them to anxiety in adults.

\section{2-kHz calls}

These calls are the most distinct from the two classes described before. Thus, 22-kHz calls, sometimes also termed low-frequency calls, are not only of much lower frequency, since they range around 18 to $32 \mathrm{kHz}$, but also of considerably longer duration (300-3000 ms). Additionally, they have a comparably simple shape and often occur in bouts of 3-5 calls, with sound pressures higher than those of the previously described $40-$ and $50-\mathrm{kHz}$ classes. Dur-

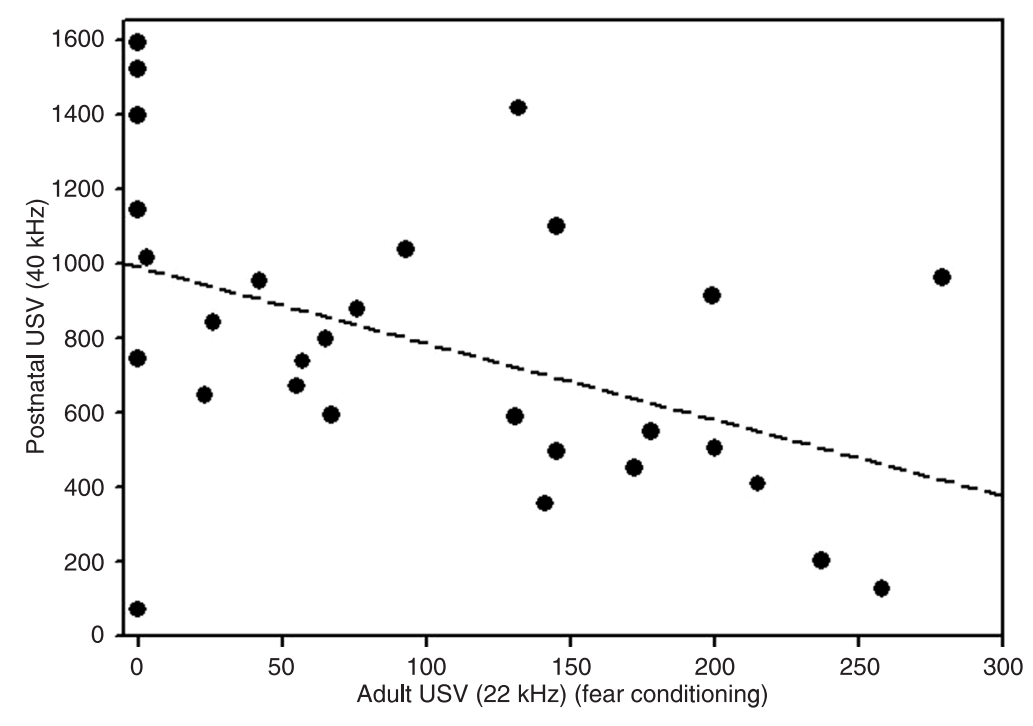

Figure 3. Relationship between ultrasonic vocalizations (USV) emitted in infancy and adulthood. Scatter plot depicting individual relationships between pup USV (40-kHz calls) emitted during a 10-min test of isolation and adult USV displayed in a fear-conditioning paradigm $(22-\mathrm{kHz}$ calls during the conditioned/unconditioned stimulus-phase of the conditioning day). Pup and adult calling were negatively correlated with each other $(r=-429, P=0.023)$. Data are taken from Wöhr and Schwarting (Behav Neurosci 2008; 122: 310-330). 
ing such 22-kHz calls, rats are usually in an immobile but tense posture (for example freezing) and they emit their calls - preceded by short inspiration - during a rather long phase of expiration (37), paralleled by increases in heart rate and blood pressure (38-40).

Juvenile and adult rats emit 22-kHz calls in various situations (for a review, see Ref. 8), most of which have an evident aversive component: when exposed to a predator $(41,42)$, during intermale social defeat, probably as an intraspecific submissive posture $(43,44)$, during fear learning (usually with footshock) and later conditioned expression, including cue- and context-dependent fear conditioning $(4,12,21,45-$ 47 ), active avoidance learning (48), when acoustically startled (49), especially during opiate and cocaine withdrawal $(50,51)$, or when subjected to aversive handling (52), or air-puff (53). Also, 22-kHz calls have been studied in pain models such as arthritic, formalin-induced or muscular pain, but it is debated whether these ultrasonic signals provide information additional to that of audible ones (for a review, see Ref. 54). In contrast, 22-kHz calls were not observed in the elevated plus-maze (47), which is a landmark test in anxiety research, for example with respect to drug testing. Possibly, the elevated plus-maze is less aversive or involves a different kind of aversiveness for the rat than the above mentioned tests, that is, the emotional state induced may differ quantitatively and/or qualitatively from that in a fear-conditioning paradigm, for example, including the question of fear versus anxiety, or proximal versus distal threats. Finally, males appear to emit 22-kHz calls during the post-ejaculatory period (55). This latter phenomenon will not be addressed any further here, since it has not received much scientific attention and is therefore not well understood.

In general, it is assumed that 22-kHz calls a) reflect or depend on a state of anxiety, and b) can have a communicative function, in the sense of an acoustic defensive mechanism. With respect to their communicative side, 22$\mathrm{kHz}$ calls can serve as threat or pacification signals in intra- or interspecies encounters, reducing the likelihood of harmful aggression. Furthermore, these calls can also serve as alarm calls to warn conspecifics about external danger $(8,41,42)$, eliciting behavioral inhibition or flight in the recipients. Clearly, both functions are of considerable survival value for this species.

In line with an alarm function, it was shown that aversive 22-kHz USV can elicit defensive behaviors like behavioral inhibition in the recipient $(5,8,42,56-59)$, whereas $50-\mathrm{kHz}$ USV induce exploratory behavior (5) (Figure 4). Such effects were observed in semi-natural social environments, like the well-known visible burrow system, where calls leading to freezing, flight, and risk assessment were induced by cat exposure (42), but also in individual test situations, where calls were selectively presented to single rats by means of the playback technique $(5,8,56-58)$. It should be noted, however, that the kind and degree of behavioral response is variable, and may differ depending on various factors such as the type of testing situation, including its social aspects (8), features of the ultrasonic stimuli, especially their sound pressure, the rat strain, the receiver's experience, etc. For example, some studies found only moderate behavioral inhibition, but no freezing (60-62), despite clear neuronal changes in brain areas related to motivation and emotion $(61,62)$. Thus, playback of $22-\mathrm{kHz}$ calls can lead to specific activations in brain areas implicated in the processing of anxiety and defense such as the amygdala (lateral, basolateral), the adjacent perirhinal cortex, and the PAG, as shown by means of labeling immediate early genes in activated neurons or by local single-unit recordings. These patterns were distinguishable from those in response to appetitive $50-\mathrm{kHz}$ calls both with respect to their precise anatomical location and/or to the direction of change in local neural activity (8,61-63). The fact that neural activations can be measured without overt behavioral changes in parallel indicates that the subject under study clearly perceives the 22-kHz signals and processes them in limbic brain parts, but does not show a defensive response, presumably be-

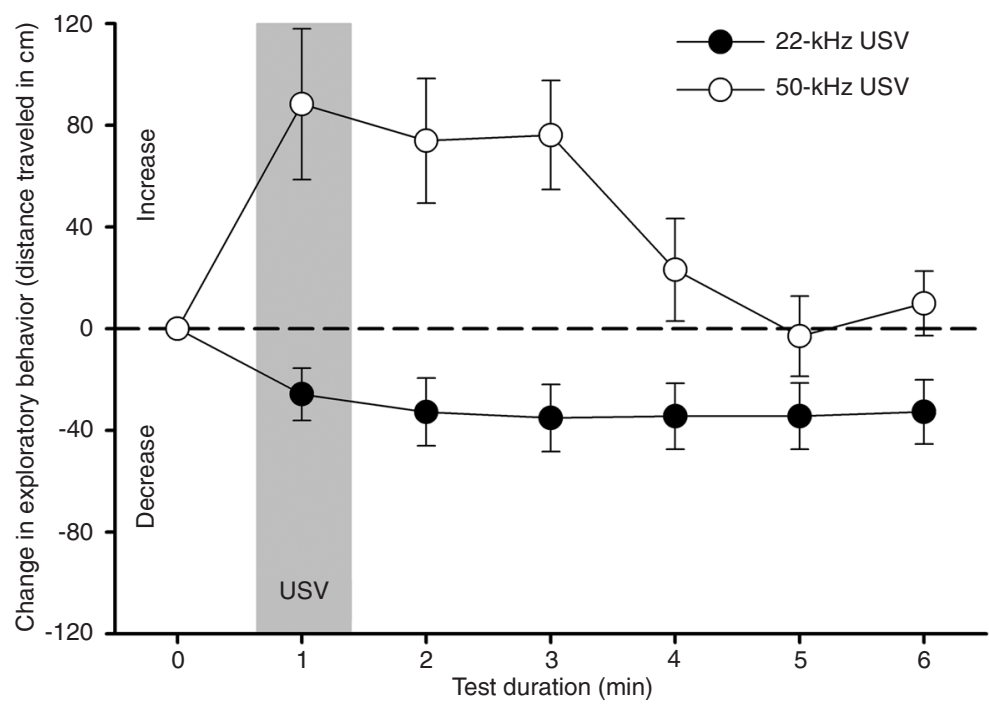

Figure 4. Behavioral changes in response to playback of 50- and 22-kHz ultrasonic vocalizations (USV). Induction of exploratory behavior by playback of 50kHz USV (white circles; $N=12$ ) and induction of behavioral inhibition by playback of 22-kHz USV (black circles; $N=12$ ), shown as distance traveled in centimeters $(\mathrm{cm})$. USV were played back for $1 \mathrm{~min}$ (highlighted in gray). Behavioral activity in the minute before playback of USV was used as baseline. Male Wistar rats at the age of 25 days were used. Data are taken from Wöhr and Schwarting (PLoS One 2007; 2: e1365). 
cause the testing conditions in general were not sufficiently aversive, or because the subject did not have previous critical aversive experience. Thus, rats may be prepared to associate $22-\mathrm{kHz}$ calls with aversiveness but may need to actually acquire their affective valence (for empirical evidence, see Refs. 60,64). This acquisition seems to occur through associative learning, with the perirhinal cortex serving as a possible "neural template" responsible for such biological preparedness $(8,64)$.

The other hypothesis, namely that 22-kHz calls reflect or depend on a state of fear and/or anxiety, is surely propelled by the hope that 22-kHz calls might serve as a gauge of the rat's presumptive subjective state, and that this behavioral information may go beyond that of conventional and usually visibly overt measures, like freezing, flight or avoidance. Although this theoretical issue remains highly problematic since we can only speculate whether rats have subjective emotional states that they can experience (mainly because we have no emotional self-report like in humans), there are several good reasons to assume that 22-kHz calls can actually serve to study anxiety, especially when anxiety is considered as a defense mechanism (and not so much as a subjective experience).

For one, and as outlined above, 22-kHz calls occur especially in aversive or threatening situations, that is, when fear and anxiety usually are appropriate states or responses. Fear conditioning is one of them, and $22-\mathrm{kHz}$ calls have been studied repeatedly there both in context- or cue-dependent versions of the paradigm. Typically, 22$\mathrm{kHz}$ calls do not occur during experience of the aversive electric shock itself (and when the rat is trying to escape it), but - and after only a few such experiences - during subsequent inter-trial intervals. Also, cue-conditioned rats do not vocalize in response to the mere context (4), or require several conditioning sessions to emit such a response (39). Importantly, USV rates, unlike freezing, are usually much lower during retention tests than during the initial conditioning trial $(4,37)$ indicating that USV should not be used as a reliable index of an aversive memory, especially not in context-dependent paradigms (37).

Call likelihood and rate can depend on the degree of aversiveness, with a ceiling effect in case of high shock intensities (4). Also, USV rates in cue-conditioning paradigms are typically high during the inter-trial intervals, whereas rats tend to stop calling during the subsequent conditioned stimulus (CS) phases $(4,38,46)$, as if the rat experienced the threat as imminent during CS exposure, which may lead to a suppression of calling. Accordingly, Jelen et al. (46) suggested that USV might be useful to differentiate between fear and anxiety in rats, based on the assumption that the CS, which signals the subsequent unavoidable electric shock, induces acute fear, whereas the inter-trial interval, where the threat may be more distal, evokes anxiety. Such a distinction cannot be provided by the conventional measure of freezing, since freezing rates are often similar during the two states. In general, however, the emission of $22-\mathrm{kHz}$ calls is linked to freezing in fear conditioning paradigms, since call rates and freezing durations were found to be correlated with each other (4), but it should be noted that vocalization in a fear conditioning paradigm is less likely than freezing or autonomic activation. In other words, not every rat that shows freezing or increased heart rate in response to an aversive CS also emits 22-kHz calls. This pattern indicates that $22-\mathrm{kHz}$ calls cannot be taken as simple readouts of an emotional state, like anxiety, fear, or distress. These states might be considered as necessary, but not sufficient requirements for the occurrence of $22-\mathrm{kHz}$ calls in aversive situations, which seem to depend also on other, yet not fully understood mechanisms. The following section aims to summarize what we know about them.

\section{Role of individual and social factors}

Our own laboratory experience, but also that of many other research groups, shows that the likelihood of emitting, or the number of actually emitted $22-\mathrm{kHz}$ calls differs substantially between rats. Wide variability in calling also holds for juvenile and adult appetitive $50-\mathrm{kHz}$ calls (e.g., $12,13)$, whereas variability is often lower in pup $40-\mathrm{kHz}$ calls. Lower variability in pups might be due to the high survival value of isolation-induced calls for each subject, whereas juvenile and adult calls may be determined by a number of experiential and situative factors, which differ between subjects and may therefore explain their variability. The precise reasons for this variability, however, are not understood, although variability is often taken for granted. Also, some publications report variability within the subject sample or indicate that animals falling below a certain cut-off criterion of calling, for example in an initial screening test, are discarded from further analysis, whereas other studies do not provide such sample information at all. Overall, inter-individual variability in 22-kHz calling is an apparently common but neglected aspect of this kind of research.

When looking at studies that report variability (see also Ref. 37), or that attempt to explain it, the following patterns become apparent: in cue-dependent fear-conditioning experiments where we used electric shock levels sufficient to elicit freezing in most, if not all, rats (for example, 0.5 $\mathrm{mA} / 0.5 \mathrm{~s}), 22-\mathrm{kHz}$ calls were emitted only by a subset of them. The percentage was usually highest during the conditioning trial and declined substantially in subsequent test trials (i.e., with CS+ but not shock). When comparing the percentages of "responders" during testing between studies, their fraction roughly ranged around $50 \%$ of otherwise unselected subject samples: 10/18 (21), 9/20 (47), $5 / 12$ (59), and $3 / 7$ (4). In our most recent study (65), the percentage of responders was even lower (5/44), but this may have been due to several methodological differences 
compared to our previous study.

The fact that fear-conditioned freezing and 22-kHz calls can be dissociated is actually not surprising: It is well known (see, e.g., Ref. 66) that different fear-related responses need not be activated to the same extent, since they are governed by partly different neuronal mechanisms of motor output. Also, a decoupling of freezing behavior and USV might reflect their different adaptive values: while the former is a kind of defensive behavior, which reduces the likelihood of being detected by a predator, the production of $22-\mathrm{kHz}$ USVs may serve a communicative alarm function to warn conspecifics about external danger $(8,42,59)$. Since 22$\mathrm{kHz}$ alarm calls can be detected by some predators, there might be a conflict between producing alarm calls to warn conspecifics and remaining silent to avoid being detected by predators. That is, similar to freezing behavior, remaining silent could also represent an adaptive response, at least for some subjects of a given population.

Currently, there is some evidence about the features of such a population: we had shown in male Wistar rats that subjects with higher trait anxiety, as screened in a preceding test in the elevated plus-maze, were more likely to vocalize in the fear-conditioning paradigm compared to rats with low trait anxiety (47). Others (67) using air puff-induced $22-\mathrm{kHz}$ calls showed that socially dominant rats started to vocalize sooner in response to this aversive stimulation, but showed lower total call rates than subdominant rats. More calling of subdominant rats was also observed using aversive nipping of the neck (68). It would be interesting to test whether such call differences also hold in case of fear-conditioning paradigms.

Also, it is known that socially housed rats show more $22-\mathrm{kHz}$ calls than individually housed rats $(52,67-69$, but see Ref. 70). The mechanism by which housing conditions affect $22-\mathrm{kHz}$ call emission is not clear, but it was suggested that individual housing impairs the subject's defensive mechanisms, especially those which serve for intraspecific signaling in case of proximal danger (69). This may be especially critical when rats are isolated starting from weaning, that is, such subjects are probably deprived of learning to establish social hierarchies including the use of $22-\mathrm{kHz}$ calls during agonistic encounters. In this situation, 22-kHz calls are typically emitted by the "underdog", which can prevent further attacks with this submissive signaling. A lack of such social and call experience may be generalized to other situations where the aversiveness is not provided by a conspecific but by other factors of the testing situation, such as air puff or fear conditioning. Finally, it has been shown that the likelihood of emitting 22-kHz calls can be increased if rats have a prior ("traumatic") history of juvenile stress (65) or intense footshock experience (71). There is an increasing amount of data showing that the propensity to emit $22-\mathrm{kHz}$ calls in response to aversive stimuli is modulated by prior social and non-social experiences.
Finally, the fact should be addressed that differences in call emission cannot only be observed within, but also between defined subject samples. For one, gender differences were reported but these differed between studies and tests: Graham et al. (72) observed more 22-kHz calls (and more freezing) during fear conditioning in males, whereas Blanchard et al. (41) observed more calls in females kept in a social environment and with a cat as the threat stimulus. With respect to specific breeding lines, Naito et al. (73) studied Tsukuba high- versus low-emotional rats, which are derived from Wistar rats, differing in locomotor activity or freezing in a light/dark runway. They found that the percentage of rats emitting 22-kHz calls as an acute response to electric footshock was higher in high- than low-emotional rats (68 versus 47\%). Also, they found that activation of the hypothalamic-pituitary-adrenal (HPA) axis, i.e., adrenocorticotropic hormone (ACTH) and corticosterone, did not differ between vocalizing and non-vocalizing rats, that is, the differences in call emission cannot be explained in terms of the acute stress responses induced. Finally, common outbred rat strains were compared, and Walker et al. (74), using a resident/intruder paradigm, found more calls in Sprague-Dawley than Wistar rats, which also showed less submissive postures. Sprague-Dawley rats were also found to emit more 22-kHz calls during fear conditioning compared to Long-Evans rats (72), or compared to LongEvans and Wistar rats (33). Importantly, the differences in calling were not paralleled by similar ones in freezing, with Long-Evans rats showing the highest rates (33). The call differences between strains may be explained by differences in emotionality, coping styles, or defense mechanism, or by differences in aversive learning in these conditioning procedures. Interestingly, the adult calling patterns in $22-\mathrm{kHz}$ calls were not paralleled by similar ones in $40-\mathrm{kHz}$ calls of pups during isolation, where Sprague-Dawley rats showed the lowest rates (33), again supporting our hypothesis that pup vocalization should not be taken as a general read-out of anxiety.

Together, one can assume that variability of $22-\mathrm{kHz}$ calls, as emitted during aversive situations is dependent on the following factors: 1) the kind and features of the aversive or threatening stimulus, which induces an acute state of fear, 2) gender, age, and rat strain under study, 3) the subject's level of trait anxiety, and 4) its social experience and status. Research on this variability should deserve more attention in the future. For one, such research may help to understand the phenomenon of $22-\mathrm{kHz}$ calling as such, that is, the mechanism that modulates and moderates it, and secondly since this - at least partly systematic - variability might help to study and understand variability of other experimental factors: as an example, we have recently shown that rats that respond with 22-kHz calls, that is, in an anxious way, to an otherwise appetitive kind of tactile stimulation ("tickling") did not show an increase in hippocampal cell proliferations 
compared to rats of the same sample that responded with the expected $50-\mathrm{kHz}$ calls (13). Therefore, we suggested that the effect of tickling on hippocampal cell proliferation depended on an interaction between a predisposing trait and stimulation-dependent variations of the subject's affective state. If these factors had not been taken into account, that is, if the 22-kHz measure had not been applied, an effect of the experimental variable would not have been obtained. Such critical interaction might hold also for other experimental variables, for example, anxiolytic or antidepressant drug treatments.

In the following and final section, an outline will be provided of the brain areas and neuropharmacological mechanisms known to be relevant for $22-\mathrm{kHz}$ calls in rats.

\section{Brain mechanisms and pharmacology}

\section{Brain stem}

Yajima et al. (75) showed that 22-kHz calls can be elicited with electrical stimulation placed in the areas of the lateral reticular and facial nucleus of the medulla oblongata. These sites probably innervate relevant facial and laryngeal musculature and partly overlap descending noradrenergic sites (A1, A2, A5). Importantly, the sites effective for 22-kHz calls where not identical to those where audible vocalizations could be induced. Furthermore, Yajima et al. (76) found sensitive areas in midline thalamic nuclei (periventricular, reuniens, rhomboid) (see also Ref. 77), the posterior hypothalamus, and the PAG, especially in its dorsal and medial parts. Later research (78) supported the critical role of the PAG by showing that chemical stimulation by means of local kainic acid injections into the caudal or lateral PAG also induced 22-kHz calls, whereas GABAergic agonism or glutamatergic antagonism (ventrolateral PAG) reduced 22-kHz calls and freezing (the rats now actually became agitated) in a contextual fear conditioning paradigm, but left cardio-vascular activations unaffected (40). The thalamic and hypothalamic sites maintain a reciprocal anatomical interaction with the PAG, which is provided by the dorsal longitudinal fasciculus of Schütz (or periventricular fiber system). Thus, one can assume that the PAG may serve as a bottleneck-like structure, which receives call-relevant diencephalic inputs and sends the relevant action commands to medullary motoneurons.

\section{Forebrain}

The role of forebrain structures for 22-kHz calls is based largely on lesion approaches to a number of critical brain sites including cortex, amygdala, dorsal hippocampus, fornix, and nucleus accumbens $(38,77,79)$, where $22-\mathrm{kHz}$ calls, as elicited in different fear-conditioning paradigms, can be reduced or abolished. Most of these structures are known from other research on fear and anxiety mechanisms, and can be assigned to a larger aversive emotional network. Interestingly, NMDA-induced lesions in the ventromedial (infralimbic) frontal cortex did not affect shock-induced 22$\mathrm{kHz}$ calls, but reduced the number of animals vocalizing (and freezing) in response to the CS+ tone (38), indicating that unconditioned and conditioned mechanisms can be differentiated here. Also, Antoniadis and McDonald (77) showed in their comprehensive lesion study that the nucleus accumbens was the most effective site to reduce $22-\mathrm{kHz}$ calls in a contextual fear-conditioning paradigm. Importantly, this impairment was not paralleled by a deficit in avoiding the aversive environment, indicating that the effect of lesion in the nucleus accumbens was functionally selective, which adds to other current evidence showing that this nucleus is linked not only to appetitive, but also to aversive mechanisms. Finally, studies of local electrophysiology and c-Fos labeling of activated neurons showed that some of the sites critical for 22-kHz call emission are also activated when rats perceive 22-kHz calls, such as sub-nuclei in the amygdala, the PAG, and frontal cortical sites $(61,62)$. This may indicate an anatomical overlap between or integration of relevant aversive sensory inputs, on the one hand, and appropriate functional consequences there, on the other. Figure 5 summarizes the brain structures involved in the production of 22-kHz USV.

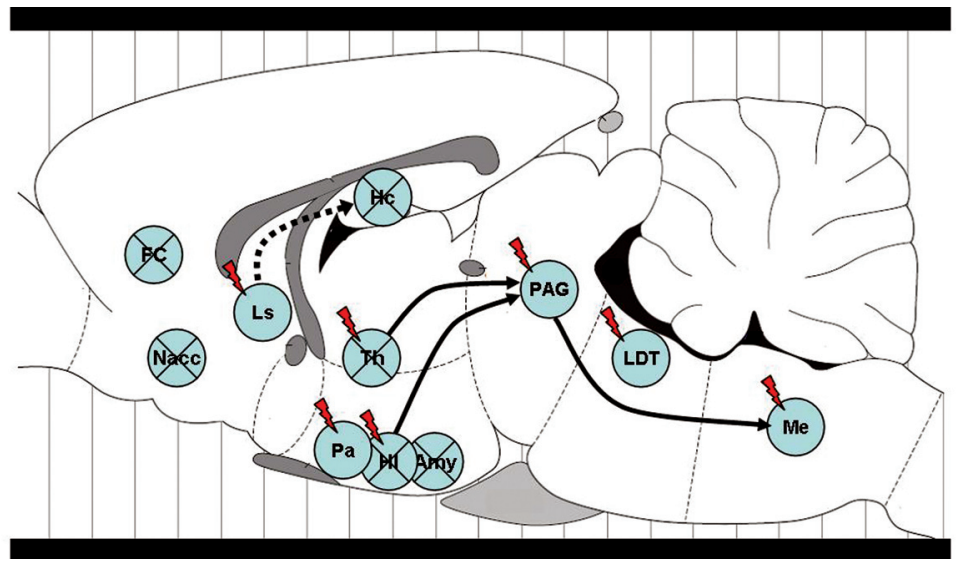

Figure 5. Brain structures involved in the production of 22-kHz ultrasonic vocalizations (USV). Brain areas where electrical or chemical stimulation (indicated by a flash symbol) induced or where lesions (indicated by an $\mathrm{X}$ ) decreased or abolished 22-kHz calls in rats. $\mathrm{FC}=$ frontal cortex (ventromedial); Nacc $=$ nucleus accumbens; $\mathrm{Ls}=$ lateral septum; $\mathrm{Pa}=$ preoptic area; $\mathrm{Amy}=$ amygdala; $\mathrm{HI}=$ hypothalamus (posterior, perifornical); $\mathrm{Hc}=$ hippocampus (dorsal); $\mathrm{Th}=$ thalamus (midline thalamic nuclei); PAG = periaqueductal gray; LDT = laterodorsal tegmental nucleus; $\mathrm{Me}=$ medulla oblongata (lateral reticular and facial nucleus). Furthermore, the fornix, lesions of which can also reduce calling, is indicated by the dashed line. 


\section{Pharmacology}

The effects of drugs on 22-kHz calls have been intensively investigated since the eighties of the last century, not the least since it was hoped that this behavioral phenomenon might serve as a readout of an internal state of anxiety, which could serve as a tool for screening clinically relevant anxiolytic drugs. In fact, a number of drugs known to have anxiolytic actions in humans can reduce or suppress $22-\mathrm{kHz}$ calls in rats, including the benzodiazepines diazepam and midazolam, 5- $\mathrm{HT}_{1 \mathrm{~A}}$ and $5-\mathrm{HT}_{2}$ agonists, 5- $\mathrm{HT}$ reuptake inhibitors, pentobarbital, a CRF1 antagonist, meprobamat, and chlordiazepoxid $(46,51,70,80-85)$. With respect to benzodiazepines, Sanchez (85) pointed out that their effects on $22-\mathrm{kHz}$ calls are actually rather variable, and high doses, for example in the case of diazepam, are sometimes required, but these also induce sedation and muscle relaxation. This effect pattern could theoretically be important: assuming that 22-kHz calls reflect stimulus-dependent rather than general anxiety, the limited effectiveness of benzodiazepines makes sense, because these drugs are not very effective clinically in panic disorders.

Furthermore, antidepressants with catecholaminergic profiles, dopamine reuptake inhibitors, as well as classical antipsychotics, were not found to be very effective as inhibitors of 22-kHz calls, whereas a low dosed D2-agonist, psychostimulants, and ethanol were (for a review, see Ref. 85). Finally, inhibitory drug effects were also obtained with intracranial injections including 5-HT agonists into the dorsal raphe nucleus (less so into the median raphe), dorsal hippocampus, and amygdala (84), substance $P$ or a neurokinin-1 agonist into the dorsal PAG $(86,87)$, or intracerebroventricular $\beta$-endorphin, dynorphin, and enkephalin (80).

Induction or potentiation of $22-\mathrm{kHz}$ calls, on the other hand, was obtained with the anxiogenic drug phenyletetrazole (46), with icv CRF (88), and with the cannabinoid agonist CP 55,940 (89), which was attributed to the fact that cannabinoids can have anxiogenic effects with higher doses (see also Ref. 86 for other drug findings). Also, it was found that exposure to environments previously paired with naloxone or $\mathrm{LiCl}$ (16), or withdrawal from treatment with potentially addictive drugs like cocaine and morphine $(50,51)$ can enhance the likelihood of $22-\mathrm{kHz}$ calls, for example when being acoustically startled or in response to an air puff. It was assumed that such effects reflect an aversive affective state (distress). This state can be attenuated by renewed drug access, thereby supporting a vicious cycle

\section{References}

1. Brudzynski SM. Handbook of mammalian vocalization. An integrative neuroscience approach. Amsterdam: ElsevierAcademic Press; 2010.

2. Anderson JW. The production of ultrasonic sounds by of drug dependency (51).

Finally, Brudzynski (see, e.g., Ref. 90) identified brain sites (laterodorsal tegmental nucleus, anteromedial hypothalamus, preoptic area, lateral septum, medial thalamus) where stimulation of cholinergic (muscarinic) receptors, usually by means of carbachol, induces $22-\mathrm{kHz}$ calls that are paralleled by other defensive features like freezing, and activation of the autonomic nervous system, that is, these drug challenges had profound effects on the state of the whole organism. These outcomes, which resemble previous ones found in cats (for details, see Ref. 90), are attributed to stimulation of an ascending cholinergic projection (termed "medial cholinoceptive vocalizations strip") originating in the laterodorsal tegmental nucleus. Activation of this strip might serve to induce a complex state of defensiveness, which is critical for alarm responses to dangerous stimuli.

\section{Conclusions}

At present, research on rat USV encompasses a time period of more than 50 years since its discovery. This research has provided a number of interesting findings not only for a better understanding of the subject's behavior, but also of its underlying neural mechanisms. With respect to fear and anxiety, $40-\mathrm{kHz}$ calls of pups and 22-kHz calls of juvenile or adult rats have been intensively studied both with respect to affect and to communication. It is clear that these signals, especially the $22-\mathrm{kHz}$ ones, are related to anxiety and fear, and may be part of the rat's defense mechanisms, but readers and researchers should interpret these phenomena with great caution, especially when it comes to the question whether USV can be taken as "readouts" of a subjective internal state. Apart from this theoretical aspect, which requires further elaboration in the future, rat USV provide a rather unique behavioral approach. This approach currently attracts increasing scientific attention, which with time will lead us to fully understand how these calls are controlled in the brain of the sender, how they are processed in the receiver, and why call likelihood differs so substantially between subjects.

\section{Acknowledgments}

Research supported by grants from the Deutsche Forschungsgemeinschaft (Schw 559/8-3, 10-1) and the Brazilian/German scientific exchange program PROBRAL funded by CAPES and DAAD. 
4. Wohr M, Borta A, Schwarting RK. Overt behavior and ultrasonic vocalization in a fear conditioning paradigm: a doseresponse study in the rat. Neurobiol Learn Mem 2005; 84: 228-240.

5. Wohr M, Schwarting RK. Ultrasonic communication in rats: can playback of $50-\mathrm{kHz}$ calls induce approach behavior? PLoS One 2007; 2: e1365.

6. Knutson B, Burgdorf J, Panksepp J. Ultrasonic vocalizations as indices of affective states in rats. Psychol Bull 2002; 128: 961-977.

7. Portfors CV. Types and functions of ultrasonic vocalizations in laboratory rats and mice. J Am Assoc Lab Anim Sci 2007; 46: 28-34.

8. Wöhr M, Schwarting RKW. Activation of limbic system structures by replay of ultrasonic vocalization in rats. Chapter 4.2. In: Brudzynski SM (Editor), Handbook of mammalian vocalization. An integrative neuroscience approach. Amsterdam: Elsevier-Academic Press; 2010. p 113-124.

9. Roberts $\mathrm{LH}$. The rodent ultrasound production mechanism. Ultrasonics 1975; 13: 83-88.

10. Knutson B, Burgdorf J, Panksepp J. Anticipation of play elicits high-frequency ultrasonic vocalizations in young rats. J Comp Psychol 1998; 112: 65-73.

11. Panksepp J, Burgdorf J. 50-kHz chirping (laughter?) in response to conditioned and unconditioned tickle-induced reward in rats: effects of social housing and genetic variables. Behav Brain Res 2000; 115: 25-38.

12. Schwarting RK, Jegan N, Wohr M. Situational factors, conditions and individual variables which can determine ultrasonic vocalizations in male adult Wistar rats. Behav Brain Res 2007; 182: 208-222.

13. Wohr M, Kehl M, Borta A, Schanzer A, Schwarting RK, Hoglinger GU. New insights into the relationship of neurogenesis and affect: tickling induces hippocampal cell proliferation in rats emitting appetitive $50-\mathrm{kHz}$ ultrasonic vocalizations. Neuroscience 2009; 163: 1024-1030.

14. Sales GD. Ultrasound and mating behaviour in rodents with some observations on other behavioural situations. $J$ Zool 1972; 168: 149-164.

15. Burgdorf J, Knutson B, Panksepp J. Anticipation of rewarding electrical brain stimulation evokes ultrasonic vocalization in rats. Behav Neurosci 2000; 114: 320-327.

16. Burgdorf J, Knutson B, Panksepp J, Ikemoto S. Nucleus accumbens amphetamine microinjections unconditionally elicit 50-kHz ultrasonic vocalizations in rats. Behav Neurosci 2001; 115: 940-944.

17. Knutson B, Burgdorf J, Panksepp J. High-frequency ultrasonic vocalizations index conditioned pharmacological reward in rats. Physiol Behav 1999; 66: 639-643.

18. Natusch $C$, Schwarting RK. Using bedding in a test environment critically affects $50-\mathrm{kHz}$ ultrasonic vocalizations in laboratory rats. Pharmacol Biochem Behav 2010; 96: 251-259.

19. Hofer MA. Multiple regulators of ultrasonic vocalization in the infant rat. Psychoneuroendocrinology 1996; 21: 203-217.

20. Allin JT, Banks EM. Functional aspects of ultrasound production by infant albino rats (Rattus norvegicus). Anim Behav 1972; 20: 175-185.

21. Wohr M, Schwarting RK. Maternal care, isolation-induced infant ultrasonic calling, and their relations to adult anxietyrelated behavior in the rat. Behav Neurosci 2008; 122: 310330.

22. Gardner CR. Distress vocalization in rat pups. A simple screening method for anxiolytic drugs. J Pharmacol Methods 1985; 14: 181-187.

23. Insel TR, Hill JL, Mayor RB. Rat pup ultrasonic isolation calls: possible mediation by the benzodiazepine receptor complex. Pharmacol Biochem Behav 1986; 24: 1263-1267.

24. Branchi I, Santucci D, Alleva E. Ultrasonic vocalisation emitted by infant rodents: a tool for assessment of neurobehavioural development. Behav Brain Res 2001; 125: 49-56.

25. Brunelli SA. Selective breeding for an infant phenotype: rat pup ultrasonic vocalization (USV). Behav Genet 2005; 35: 53-65.

26. Insel TR, Hill JL. Infant separation distress in genetically fearful rats. Biol Psychiatry 1987; 22: 786-789.

27. Naito $\mathrm{H}$, Inoue $\mathrm{M}$, Makino J. Ultrasonic isolation calls in genetically high- and low-emotional rat pups. Exp Anim 2000; 49: 289-294.

28. Wigger A, Loerscher $P$, Weissenbacher $P$, Holsboer $F$, Landgraf $R$. Cross-fostering and cross-breeding of $\mathrm{HAB}$ and LAB rats: a genetic rat model of anxiety. Behav Genet 2001; 31: 371-382.

29. Blumberg MS, Sokoloff G. Do infant rats cry? Psychol Rev 2001; 108: 83-95.

30. Middlemis-Brown JE, Johnson ED, Blumberg MS. Separable brainstem and forebrain contributions to ultrasonic vocalizations in infant rats. Behav Neurosci 2005; 119: 1111-1117.

31. Hofer MA, Shair HN. Ultrasonic vocalization by rat pups during recovery from deep hypothermia. Dev Psychobiol 1992; 25: 511-528.

32. Schwarting RKW, Pawlak CR. Behavioral neuroscience in the rat: Taking the individual into account. Meth Find Exp Clin Pharmacol 2004; 26: 17-22.

33. Natusch C, Schwarting RKW. Strain and sex differences in motivation-related ultrasonic vocalizations in rats. Poster (No. 195.19). Annual Meeting of the Society for Neuroscience. 2009 Oct 17-21; Chicago.

34. Searle LV. The organization of hereditary maze-brightness and maze-dullness. Genetic Psychol Monogr 1949; 39: 279325.

35. Muller J, Brunelli S, Shair H. Rat infant isolation vocalizations and their modulation by social cues as a model of expression of infantile emotionality. Chapter 6.4. In: Brudzynski SM (Editor), Handbook of mammalian vocalization. An integrative neuroscience approach. Amsterdam: Elsevier-Academic Press; 2010. p 227-240.

36. Wiedenmayer CP, Barr GA. Developmental changes in responsivity to threat are stimulus-specific in rats. Dev Psychobiol 2001; 39: 1-7.

37. Hegoburu C, Shionoya K, Garcia S, Messaoudi B, Thevenet M, Mouly AM. The RUB Cage: Respiration-Ultrasonic Vocalizations-Behavior Acquisition Setup for Assessing Emotional Memory in Rats. Front Behav Neurosci 2011; 5: 25.

38. Frysztak RJ, Neafsey EJ. The effect of medial frontal cortex lesions on respiration, "freezing", and ultrasonic vocalizations during conditioned emotional responses in rats. Cere $b$ Cortex 1991; 1: 418-425.

39. Antoniadis EA, McDonald RJ. Discriminative fear conditioning to context expressed by multiple measures of fear in the rat. Behav Brain Res 1999; 101: 1-13.

40. Walker P, Carrive P. Role of ventrolateral periaqueductal gray neurons in the behavioral and cardiovascular responses to contextual conditioned fear and poststress recovery. $\mathrm{Neu}$ roscience 2003; 116: 897-912. 
41. Blanchard RJ, Agullana R, McGee L, Weiss S, Blanchard DC. Sex differences in the incidence and sonographic characteristics of antipredator ultrasonic cries in the laboratory rat (Rattus norvegicus). J Comp Psychol 1992; 106: 270277.

42. Blanchard RJ, Blanchard DC, Agullana R, Weiss SM. Twenty-two $\mathrm{kHz}$ alarm cries to presentation of a predator, by laboratory rats living in visible burrow systems. Physiol Behav 1991; 50: 967-972.

43. Thomas DA, Takahashi LK, Barfield RJ. Analysis of ultrasonic vocalizations emitted by intruders during aggressive encounters among rats (Rattus norvegicus). J Comp Psychol 1983; 97: 201-206.

44. Sales GD. Ultrasound and aggressive behaviour in rats and other small mammals. Anim Behav 1972; 20: 88-100.

45. Choi JS, Brown TH. Central amygdala lesions block ultrasonic vocalization and freezing as conditional but not unconditional responses. J Neurosci 2003; 23: 8713-8721.

46. Jelen P, Soltysik S, Zagrodzka J. 22-kHz ultrasonic vocalization in rats as an index of anxiety but not fear: behavioral and pharmacological modulation of affective state. Behav Brain Res 2003; 141: 63-72.

47. Borta A, Wohr M, Schwarting RK. Rat ultrasonic vocalization in aversively motivated situations and the role of individual differences in anxiety-related behavior. Behav Brain Res 2006; 166: 271-280.

48. Ludwig V, Schwarting RK. Neurochemical and behavioral consequences of striatal injection of 5,7-dihydroxytryptamine. J Neurosci Methods 2007; 162: 108-118.

49. Kaltwasser MT. Startle-inducing acoustic stimuli evoke ultrasonic vocalization in the rat. Physiol Behav 1990; 48: 13-17.

50. Vivian JA, Miczek KA. Ultrasounds during morphine withdrawal in rats. Psychopharmacology 1991; 104: 187-193.

51. Covington HE III, Miczek KA. Vocalizations during withdrawal from opiates and cocaine: possible expressions of affective distress. Eur J Pharmacol 2003; 467: 1-13.

52. Brudzynski SM, Ociepa D. Ultrasonic vocalization of laboratory rats in response to handling and touch. Physiol Behav 1992; 52: 655-660.

53. Knapp DJ, Pohorecky LA. An air-puff stimulus method for elicitation of ultrasonic vocalizations in rats. $J$ Neurosci Methods 1995; 62: 1-5.

54. Zhang XJ, Zhang TW, Hu SJ, Xu H. Behavioral assessments of the aversive quality of pain in animals. Neurosci Bull 2011; 27: 61-67.

55. Barfield RJ, Geyer LA. Sexual behavior: ultrasonic postejaculatory song of the male rat. Science 1972; 176: 13491350.

56. Brudzynski SM, Chiu EM. Behavioural responses of laboratory rats to playback of $22 \mathrm{kHz}$ ultrasonic calls. Physiol Behav 1995; 57: 1039-1044.

57. Burman OHP, llyat A, Jones G, Mendl M. Ultrasonic vocalizations as indicators of welfare for laboratory rats (Rattus norvegicus). Appl Anim Behav Sci 2007; 104: 116-129.

58. Sales GD. The effect of $22 \mathrm{kHz}$ calls and artificial $38 \mathrm{kHz}$ signals on activity in rats. Behav Proc 1991; 24: 83-93.

59. Wöhr M, Schwarting RKW. Ultrasonic calling during fear conditioning in the rat: no evidence for an audience effect. Anim Behav 2008; 76: 749-760.

60. Bang SJ, Allen TA, Jones LK, Boguszewski P, Brown TH. Asymmetrical stimulus generalization following differential fear conditioning. Neurobiol Learn Mem 2008; 90: 200216.

61. Sadananda M, Wohr M, Schwarting RK. Playback of $22-\mathrm{kHz}$ and $50-\mathrm{kHz}$ ultrasonic vocalizations induces differential c-fos expression in rat brain. Neurosci Lett 2008; 435: 17-23.

62. Parsana AJ, Li N, Brown TH. Positive and negative ultrasonic social signals elicit opposing firing patterns in rat amygdala. Behav Brain Res 2012; 226: 77-86.

63. Allen TA, Furtak SC, Brown TH. Single-unit responses to 22 $\mathrm{kHz}$ ultrasonic vocalizations in rat perirhinal cortex. Behav Brain Res 2007; 182: 327-336.

64. Endres T, Widmann K, Fendt M. Are rats predisposed to learn $22 \mathrm{kHz}$ calls as danger-predicting signals? Behav Brain Res 2007; 185: 69-75.

65. Yee N, Schwarting RK, Fuchs E, Wohr M. Juvenile stress potentiates aversive $22-\mathrm{kHz}$ ultrasonic vocalizations and freezing during auditory fear conditioning in adult male rats. Stress 2012 (in press).

66. Kosten TA, Lee HJ, Kim JJ. Early life stress impairs fear conditioning in adult male and female rats. Brain Res 2006; 1087: 142-150.

67. Pohorecky LA. Psychosocial stress and chronic ethanol ingestion in male rats: effects on elevated plus maze behavior and ultrasonic vocalizations. Physiol Behav 2008; 94: 432447.

68. Inagaki $\mathrm{H}$, Kuwahara M, Kikusui T, Tsubone $\mathrm{H}$. The influence of social environmental condition on the production of stressinduced $22 \mathrm{kHz}$ calls in adult male Wistar rats. Physiol Behav 2005; 84: 17-22.

69. Nunes Mamede Rosa ML, Nobre MJ, Ribeiro OA, Brandão $\mathrm{ML}$. Isolation-induced changes in ultrasonic vocalization, fear-potentiated startle and prepulse inhibition in rats. Neuropsychobiology 2005; 51: 248-255.

70. Tomazini FM, Reimer A, Albrechet-Souza L, Brandão ML. Opposite effects of short- and long-duration isolation on ultrasonic vocalization, startle and prepulse inhibition in rats. J Neurosci Methods 2006; 153: 114-120.

71. Chen X, Li Y, Li S, Kirouac GJ. Early fear as a predictor of avoidance in a rat model of post-traumatic stress disorder. Behav Brain Res 2012; 226: 112-117.

72. Graham LK, Yoon T, Lee HJ, Kim JJ. Strain and sex differences in fear conditioning: $22 \mathrm{kHz}$ ultrasonic vocalizations and freezing in rats. Psychol Neurosci 2009; 2: 219-225.

73. Naito H, Inoue M, Suzuki Y, Tohei A, Watanabe G, Taya K, et al. Ultrasonic vocalization responses in genetically high- and low-emotional rats. Exp Anim 2012; 50: 285-291.

74. Walker FR, Naicker S, Hinwood M, Dunn N, Day TA. Strain differences in coping behaviour, novelty seeking behaviour, and susceptibility to socially conditioned fear: a comparison between Wistar and Sprague Dawley rats. Stress 2009; 12: 507-516.

75. Yajima $\mathrm{Y}$, Hayashi $\mathrm{Y}$, Yoshii N. The midbrain central gray substance as a highly sensitive neural structure for the production of ultrasonic vocalization in the rat. Brain Res 1980; 198: 446-452.

76. Yajima Y, Hayashi Y, Yoshii N. Identification of ultrasonic vocalization substrates determined by electrical stimulation applied to the medulla oblongata in the rat. Brain Res 1981; 229: 353-362.

77. Antoniadis EA, McDonald RJ. Fornix, medial prefrontal cortex, nucleus accumbens, and mediodorsal thalamic nucleus: roles in a fear-based context discrimination task. Neurobiol 
Learn Mem 2006; 85: 71-85

78. Depaulis A, Keay KA, Bandler R. Longitudinal neuronal organization of defensive reactions in the midbrain periaqueductal gray region of the rat. Exp Brain Res 1992; 90: 307-318

79. Antoniadis EA, McDonald RJ. Amygdala, hippocampus and discriminative fear conditioning to context. Behav Brain Res 2000; 108: 1-19.

80. Tonoue T, Ashida $\mathrm{Y}$, Makino H, Hata H. Inhibition of shockelicited ultrasonic vocalization by opioid peptides in the rat: a psychotropic effect. Psychoneuroendocrinology 1986; 11: 177-184.

81. De Vry J, Benz U, Schreiber R, Traber J. Shock-induced ultrasonic vocalization in young adult rats: a model for testing putative anti-anxiety drugs. Eur J Pharmacol 1993; 249: 331-339.

82. Kikusui T, Takeuchi Y, Mori Y. Involvement of corticotropinreleasing factor in the retrieval process of fear-conditioned ultrasonic vocalization in rats. Physiol Behav 2000; 71: 323328.

83. Nobre MJ, Brandão ML. Analysis of freezing behavior and ultrasonic vocalization in response to foot-shocks, ultrasound signals and GABAergic inhibition in the inferior colliculus: effects of muscimol and midazolam. Eur Neuropsychopharmacol 2004; 14: 45-52.

84. Schreiber R, De Vry J. Neuronal circuits involved in the anxiolytic effects of the $5-\mathrm{HT} 1 \mathrm{~A}$ receptor agonists $8-\mathrm{OH}-\mathrm{DPAT}$ ipsapirone and buspirone in the rat. Eur J Pharmacol 1993; 249: 341-351.

85. Sanchez C. Effect of serotonergic drugs on footshockinduced ultrasonic vocalization in adult male rats. Behav Pharmacol 1993; 4: 269-277.

86. Bassi GS, Nobre MJ, de Araujo JE, Brandão ML. Anxiogenic effects of activation of NK-1 receptors of the dorsal periaqueductal gray as assessed by the elevated plus-maze, ultrasound vocalizations and tail-flick tests. Neuropeptides 2007; 41: 365-374.

87. Bassi GS, Nobre MJ, Carvalho MC, Brandão ML. Substance $P$ injected into the dorsal periaqueductal gray causes anxiogenic effects similar to the long-term isolation as assessed by ultrasound vocalizations measurements. Behav Brain Res 2007; 182: 301-307.

88. Swiergiel AH, Zhou Y, Dunn AJ. Effects of chronic footshock, restraint and corticotropin-releasing factor on freezing, ultrasonic vocalization and forced swim behavior in rats. Behav Brain Res 2007; 183: 178-187.

89. Arnold JC, Dielenberg RA, McGregor IS. Cannabinoids increase conditioned ultrasonic vocalisations and cat odour avoidance in rats: strain differences in drug-induced anxiety. Life Sci 2010; 87: 572-578.

90. Brudzynski SM. Ultrasonic calls of rats as indicator variables of negative or positive states: acetylcholine-dopamine interaction and acoustic coding. Behav Brain Res 2007; 182: 261-273. 